\title{
AN AUTOPSY CASE OF PULMONARY FUNGAL DISEASE IN NEONATE
}

\author{
YASUHIRO NAKAMURA ${ }^{1)}$, SYOJIRO OKADA ${ }^{2)}$ AND TAKEO HASHIMOTO ${ }^{3)}$ \\ Second Department of Pathology ${ }^{11}$, Department of Pediatrics, Kurume \\ University School of Medicine ${ }^{2)}$ and Department of Neonatology, St. Mary's \\ Hospital ${ }^{3)}$, Kurume, 830, Japan
}

Received for publication June 14, 1979

\begin{abstract}
An autopsy case of pulmonary fungal disease in neonate was reported in this paper. In lungs, numerous abscesses were found and spherical fungal bodies without hyphae were scattered in the abscess. These fungal disease in neonate are rare condition.
\end{abstract}

\section{INTRODUCTION}

In neonatal period, the fungal diseases are relatively rare. Among them, the candidiasis is occasionally found in gastrointestinal tract, lung and other organs. Two cases of pulmonary candidiasis out of 700 autopsy cases are found in our laboratory.

Other fungal diseases ; histoplasmosis, aspergillosis, actinomycosis and so forth, are very rare conditions. In this paper, we would report the peculiar pulmonary lesion which is composed of the abscess, giant cells and spherical fungal spores. Morphologically, these organisms are not resembled to candida, aspergillus, crytococcus and actinomyces. Although the mycological determination was not done unfortunately, it might be a uncommon type of fungal disease.

\section{A CASE REPORT}

A female infant was admitted to a hospital at forth day of age because of poor sucking, poor spontaneous activity and vomiting. She was born after a normal pregnancy, but was delivered by cesarean section, partly because of hardness of mother's uterine cervix and advanced age of her mother. Her mother was 35 years old and in the first gestation. The infant weighed $2900 \mathrm{gm}$ at birth. Amniotic fluid was turbid and umbilical cord was coiled around her neck. Soon after birth, the infant's condition seemed to be fair, but within a short period of time some symptoms were arised. After admission, hemorrhagic diathesis became marked and many petechiae were found on the abdomen, face, arms and thighs. There were also tachypnea and jaundice. She was observed in head-box. The hemorrhagic diathesis became worse and worse, then petechiae were fused. The color of skin was darty and yellowish. On seventh day, anemia and symptoms of cardiac failure appeared, and jaundice was increased. On eleventh day, she was died of acute respiratory failure.

Laboratory data on admission were as follows; the hemoglobin was 12.2 $\mathrm{g} / \mathrm{dl}$, the red blood cell count $3,380,000 /$ $\mathrm{mm}^{3}$, the white blood cell count $12,600 /$ $\mathrm{mm}^{3}$, with differential count of $60 \%$ neutrophils, $39 \%$ lymphocytes, $1 \%$ monocytes. The platelet count was $34,000 / \mathrm{mm}^{3}$, and reticulated red blood cells $6 \%$. The total bilirubin was 12.0 $\mathrm{mg} / \mathrm{dl}$ and the direct-acting bilirubin was $3.6 \mathrm{mg} / \mathrm{dl}$. 
The blood types of the baby and her parents were all type $A$. The value of C-reacting protein was increased. Blood electrolytes, cerebrospinal fluid and urinalysis were all within normal limits. Repeated bacterial cultures of blood and CSF were sterile throughout the course of the patient's illness. On several serological studies for viruses, no positive data was obtained. A routine chest roentgenogram revealed the atelectasis in the upper lobe of right lung and pneumonia-like shadows in both lungs (Fig. 1).

Clinical diagnosis was sepsis occured from undetermined cause.

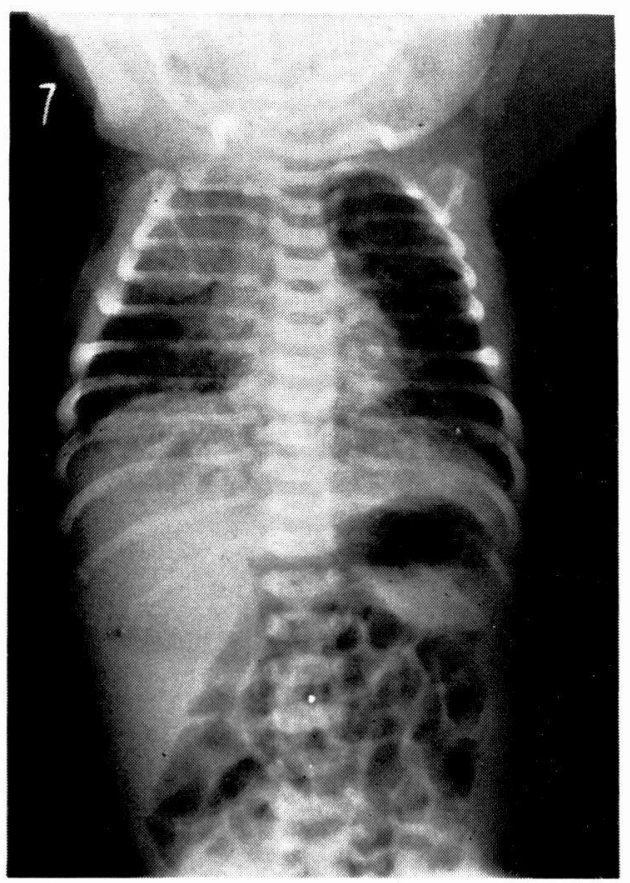

Fig. 1. Chest X-ray shows the atelectasis in the upper lobe of right lung.

\section{AUTOPSY FINDINGS}

\section{Gross examination}

The right lung weighed $70 \mathrm{gm}$. and the left $37 \mathrm{gm}$. Both lungs showed ex- tensive nodular consolidation and were covered subpleurally with multiple nodular yellow elevations of moderately firm consistency. On cut section, the lungs showed distinct firmness and surface was studded with similar yellowish nodules, some of them appearing larger because of a confluency of the nodules and these confluented lesions were prominent particularly in right upper lobe (Fig. 2). These nodular lesions had relatively sharp demarcation from adjacent pulmonary parenchyme and were not seen in any other orgas.

Hepatosplenomegaly was found, the liver weighed $191 \mathrm{gm}$, the spleen was $16 \mathrm{gm}$. The liver was enlarged and had yellwish color diffusely. Small hemorrhagic areas were found in gasrointestinal tract and pericadium. Brain was not examined.

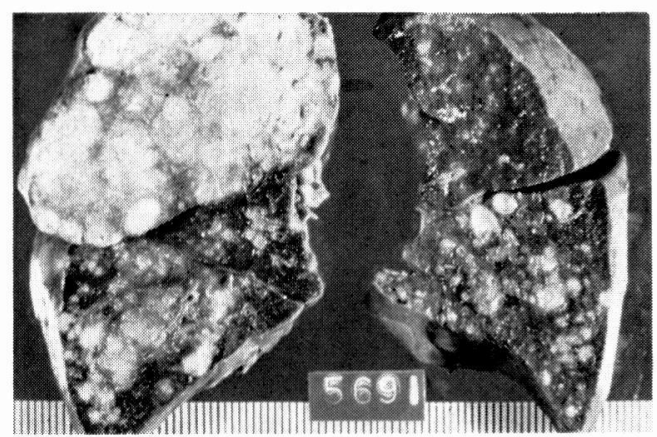

Fig. 2. Both lungs are studded with yellowish nodules, some of them are fused in right upper lobe.

\section{MICROSCOPIC EXAMINATION}

The lungs showed multiple discrete and confluent abscess composed of neutrophiles, macrophages and lymphocytes. Many multinucleated giant cells were found mainly in the periphery of the abscesses. Round or oval spherical spores were scattered throughout the lesion (Fig. 3). These organisms were 


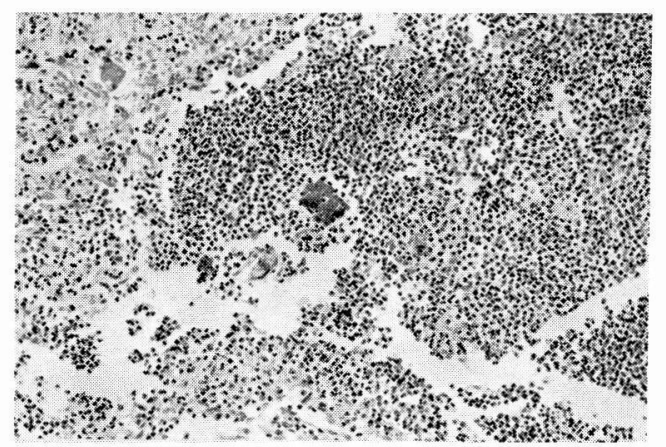

Fig. 3. Microscopically the lung shows multiple abscess with giant cells and fungal spores. H.E. $\times 100$.

gathered togather, but neither common capsules nor hyphae were found. These organisms were stainable with PAS and Glocott's method for fungus, but not stainable with Gram stain and Ziel-Nelson stain. So these organisms were confirmed to be fungi.

Some findings of other organs are as follows ; hepatosplenomegaly, bile stasis of the liver, petechiae in the gastrointestinal mucosa, extramedullary hematopoiesis in the liver, kidney, spleen, adrenal glands and pancreas, atrophy of the thymus and hyperplastic bone marrow.

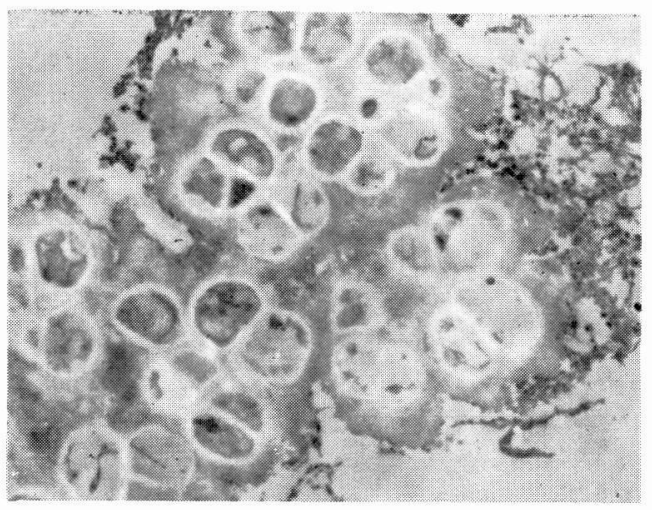

Fig. 4. Electronmicroscopic finding of fungi. $\times 12,000$.

\section{Electronmicroscopic findings}

The specimen was fixed with $10 \%$ formalin and embedded in paraffin. The paraffin embedding specimen was used to examine for electron microscopic study. The organisms were surrounded by thick cell wall and were divided into 3 or 4 small clusters (Fig. 4). Intracytoplasmic organellas were destroyed and obscure.

\section{DISCUSSION}

Diseases caused by fungi comprise a small fraction of the infections in neonatal period. Among them, candidiasis, nocardiasis, aspergillosis, histoplasmosis, coccidioidomycosis, cryptococcosis and actinomycosis are reported in neonatal or infantile period.

Candidiasis caused by Candida albicans is often secondary to maternal vaginal infection, and is found in oral cavity, esophagus, stomach and lung. Most candidiasis appear to be acquired during passage through the birth canal, although occasionally candida infection of the amniotic sac is followed by aspiration pneumonia, and organisms are often present in the gastrointestinal tract. In our study, two pulmonary cases of candidiasis are found and also present in the gastrointestinal tract. Generally speaking, the blastospores and pseudohyphae of Candida can be demonstrated clearly. Histologic reactions take a form of bronchitis and severe pneumonia. Emanual et al. (Emanuel et al., 1962) and Linhartova et al. (Linhartova et al., 1963) reported a few cases of pneumonia caused by candida in neonate, in which cases blastospores and pseudohyphae were found in the bronchioles and alveoli with polymorph leucocytes, histiocytes and occasional giant cell reaction. The giant cells contained ingested blastospores. 
Aspergillosis caused by aspergillus fumigatus is rare under an year of age. Allen and Anderson (Allen and Anderson, 1960) listed three patients under 1 year of age with this condition. The principal types of lesions include 1) non-suppurative, 2) diffuse pneumonia and suppurative, 3) saprophitic surface infections including the aspergillus mycetoma, and 4) tuberculoid form (Spencer, 1968). Aspergilli are found in tissues as a mass of corse, branching and septate hyphae. Vascular invasion is common.

Blastomycosis, coccidioidomycosis and histoplasmosis are endemic diseases and rare in neonate. In the tissue, Histoplasma capsulatum is seen as an oval yeast like body with a sharply defined, clear colorless capsule and a central dark staining chromates mass. This disease affects primarily the reticuloendotherial system, and the disease localized to the lungs is benign and heals rapidly.

Coccidioidomycosis is caused by coccidioides immitis and this disease is endemic. The pulmonary lesions produced by coccidioidomycosis are granulomas, with irregular giant cells and lymphocytes often surrounding single large endospores $20-60 \mu$ in diameter.

Christian et al. (Christian et al., 1956) reported a neonatal case.

Actinomycosis, caused by actinomyces israeli, is extremely rare in neonatal and in infantile periods. The lesion reveales the abscess containing a nonspecific purulent exudates. The organisms are consisted of a feltwork of radiating, branching filaments, often with terminal club-shaped expansion.

Cryptococcosis is caused by cryptococcus neoformans which is a yeast like, non-mycelial and spherical orga nism. Cryptococcosis produses two types of reaction; geratinous cystic abscesses, usually in brain, and focal granulomas.
Bacterial pseudomycosis resembles to the fungal infection (Spencer, 1968). In the lung, the clumps of coccal microorganisms are found in the abscess, but these organisms are not stainable with PAS and Glocott's method for fungi.

In our case, the type of histological reaction is abscess formation with giant cell reaction which is resembled to actinomycosis, candidiasis and pseudomycosis, but neither hyphae nor radiating arrangement is found. Organisms themselves are resembled to spores without envelops in coccidioidomycosis or pseudomycosis, but no granulomatous changes are found and the organisms are stainable with special stain for fungi.

It was impossible for us to deter mine the type of this organism histologically and bacteriologically, but it seems certain to be a fungus because of histochemical and electronmicroscopic findings.

It is thought that the severe pulmonary disease caused by fungi seen in our case is extremely rare condition.

\section{REFERENCES}

Allen, G. W. and Andersen, D. H. (1960). Generalized aspergillosis. Pediatrics, 26. 432-440.

Christian, J. R, Sarre, S. G., Peers, J. H, Salazar, E. and Rosario, J. (1956). Pulmonary coccidioidomycosis in a twenty one day old infant. J. Dis. Child. 92, 66-74.

Emanuel, B, Lieberman, A.D., Goldin, M. and SAnson, J. (1962). Pulmonary candidiasis in the neonatal period. J. Pediat. 61, 44-52.

Linhartova, A. and Chung, W. (1963). Bronchopulmonary moniliasis in the newborn. J. Clin. Path. 16, 56-60.

Spencer, H. (1968). Pathology of the lung. The pulmonary mycotic diseases, IInd edition. p. 267-319. 